\title{
Methodological Approach to Investigate the Behavior of Structures under Dynamic Loading Using the Multiple Criteria Decision-Making Method
}

\author{
$\underline{\text { Josef Oleksik }}^{1}$, Srivatsaa Natarajan'2), Thomas Vietor ${ }^{3)}$ \\ 1) Institut für Konstruktionstechnik, josef.oleksik@tu-braunschweig.de, Technische Universität \\ Braunschweig, Hermann-Blenk-Straße 42, 38108 Braunschweig, Germany \\ 2) Institut für Konstruktionstechnik, s.natarajan@tu-braunschweig.de, Technische Universität \\ Braunschweig, Hermann-Blenk-Straße 42, 38108 Braunschweig, Germany \\ 3) Institut für Konstruktionstechnik, t.vietor@tu-braunschweig.de, Technische Universität \\ Braunschweig, Hermann-Blenk-Straße 42, 38108 Braunschweig, Germany
}

\section{Keywords}

Material, Crash simulation, COPRAS - Multiple Criteria Decision-Making method, Design approach

\begin{abstract}
The main objective of this article is to develop a support approach for designers in the concept phase of the design process. In this paper, two different structures with metal, composite and hybrid material are investigated under dynamic loading with multiple strain rates. The optimum choice of material and structural combination is found by a methodological approach using a Multi Criteria Decision-Making Method (MCDM). It uses a step-by-step procedure in evaluating the significance of each criterion and ranks the different alternatives. This method is applied to solve various problems in the field of economics, engineering, management, cf. [1], [2], [3], [4]. In this article, COPRAS is used to rank different material and structural combinations.

Cylindrical and rectangular structures are investigated under axial and 3-point bending load. Moreover, three different constellations of material widen the comparison; they are steel and aluminum, composite material with carbon fiber and thermoplastic matrix and hybrid material, with a combination of composite and metal. The output parameters from the simulation, such as energy absorption and force, are further mathematically converted to specific energy absorption (SEA), crash-force-efficiency (CFE) and load non-uniformity (LU). PAM-Crash is used as a solver for simulation.
\end{abstract}

\section{Introduction}

In this article, we discuss the possibility of identifying different material and structural combinations depending on the boundary conditions. The goal is to build a design tool, which may be helpful for designers in the early product development. VDI2014 [5] introduces a design process of product development of composite material. In the first phase of the design process - analyzing the structural design - a design catalog would be suitable for the pre-dimensioned part. In addition to the VDI2014, a further design process exists explicitly for parts constructed as a composite. The process was published by AVK [6]. There, completely different factors and methods in designing the parts were taken into consideration. The aim of this paper is to explicate the target product of composites in a wider range. A decision-making process is involved in finding a suitable version of an application depending on the material (metal/composite) or material combination (metal + composite).

Aluminum and steel structures absorb energy in a crash situation due to plastic deformation, work hardening and heat losses. There are many more factors in composite design that need to be addressed. For this reason, composite material in general is not considered a material but as a design 
because of the complexity and variation of possibilities. Here, the various fiber orientations or laminate architectures can be understood as the variant complexity. Some of the parameters that have a significant influence in a composite design are wall thickness, fiber orientation, fiber material, fiber proportion, layer structure and some more. Furthermore, loading conditions and the manufacturing process play a major role in energy absorption, crash force efficiency and deformation of the composite material. For the case of hybrid structures, additional factors must be considered. These include, for example, the position of the composite (outside/inside), adhesive bonding between the two materials, mutual support in specific energy absorption and other. According to [7]: "Energy absorption efficiency is examined as a function of composite wall thickness, loading condition, fiber ply orientation and composite property." In [8] the fiber orientation is just represented as the function of energy absorption. Also, the Composite Materials Handbook [CMH] considers the numbers of plies and their ply angles as a given application. This book gives "strong recommendations". There are different ways to work towards the optimum combination of fiber orientation, structure, and other parameters. Crash-relevant components can either be simulated or tested experimentally. Analytical prediction of failure in crushing is not possible. A large number of authors have already tried to describe and determine the fiber orientation. [VDI2014] gives an additional proposal for fiber orientation for flat structural elements by predominant stress. Other load and stress cases are equally important in practice.

\section{Method}

\subsection{Methodological approach}

The primary investigation is aimed at developing a methodological approach to study the behavior of the structure under dynamic loading conditions. In Fig. 1 the logical process of the method is introduced. Multiple combinations of materials and geometries are tested under different loading conditions. An experiment process in the application would be very useful for authentic reasons. All parameters needed for the ranking of the method are reached by crash simulations.

In this article, COPRAS is used as a supporting method of the design tool. COPRAS is one of the very fast growing multi-criteria decision-making (MCDM) methods that can compute a large number of factors influencing the selection process. COPRAS can be efficiently applied to any type of a real-time material selection problem involving any number of qualitative and quantitative criteria, and any number of alternatives [3].

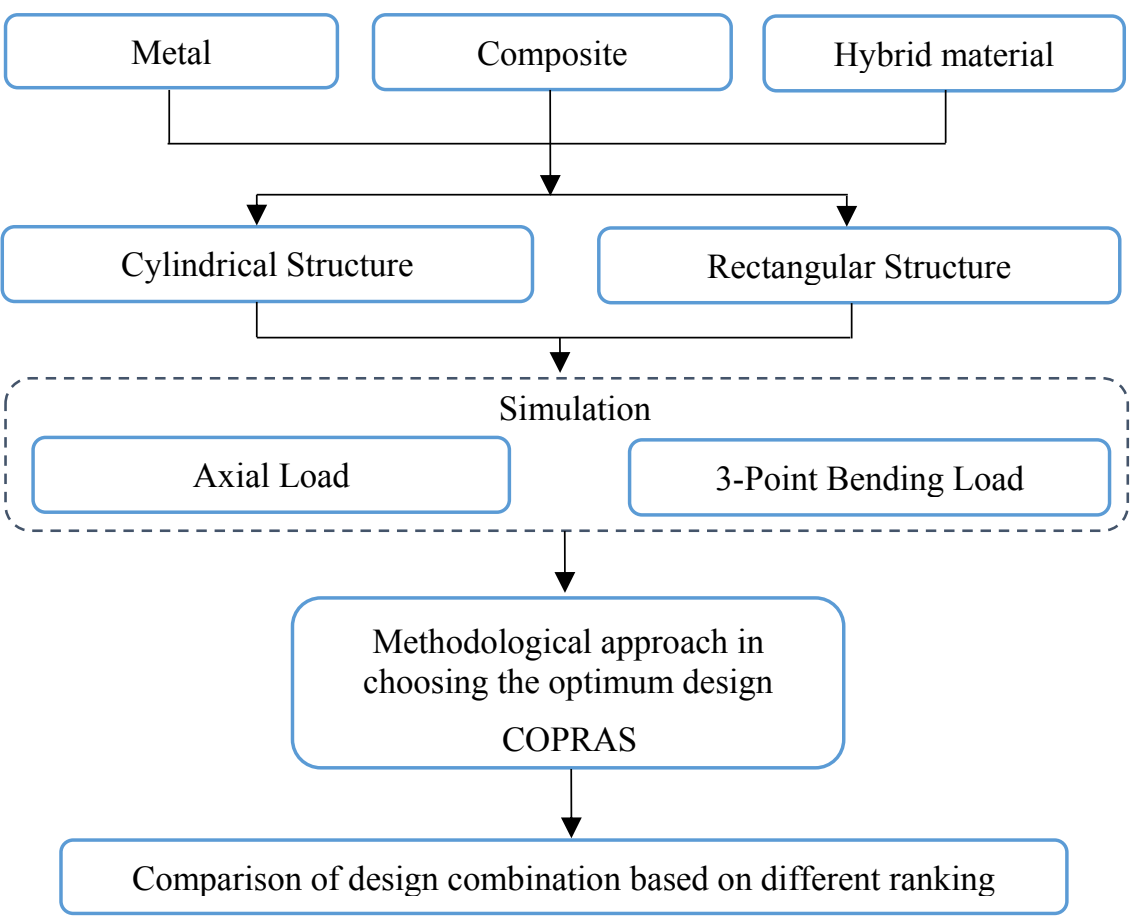

Figure 1: Methodological approach for choosing the suitable combination using COPRAS 
COPRAS decides what the optimum choice from a different set of alternatives would be. Material properties, such as specific energy absorption, crash force efficiency and load non-uniformity, are investigated. These performance indicators are weighted based on the level of importance for different alternatives. The maximum force (Fig. 2) should not show any great differences in relation to the average force. This ratio describes the crash-force-efficiency (CFE) where the average force is divided by the maximum force and should be as high as possible. The load non-uniformity is the ratio of variation $\Delta \mathrm{F}_{\text {avg }}$ and the maximum force. This ratio should be as small as possible. The area of energy absorption $\mathrm{W}_{\text {abs }}$ is to be seen under the force curve. The force profile in the range after the maximum force is different for metal and composite. Metal usually has a folding form, which causes a punctual plastic deformation. In this case, the energy absorption can be efficiently occluded only at certain points. In case of a composite, it is a smooth force process. In case of a crash box, the great advantage is represented by more uniformed stress affecting the passengers. Fig. 2 reports a typical force-displacement curve with the most important parameters for a crash part.

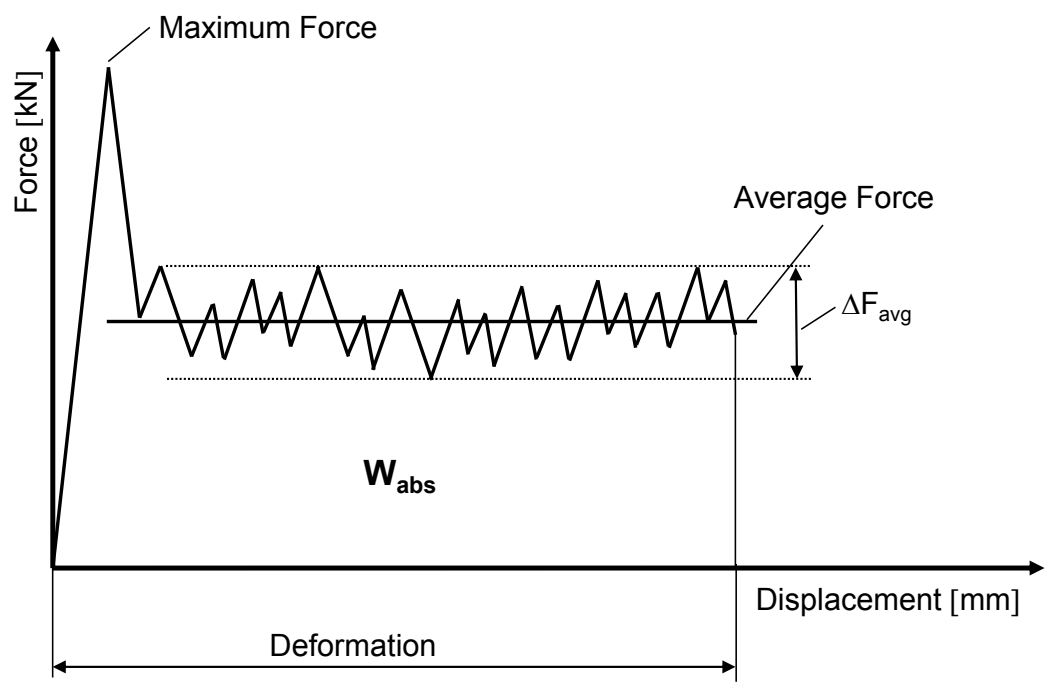

Figure 2: A typical force-displacement diagram of axial load

As an example, the force-deformation diagram of a composite material and a cylinder profile by axial loading is displayed in Fig. 3 (left).
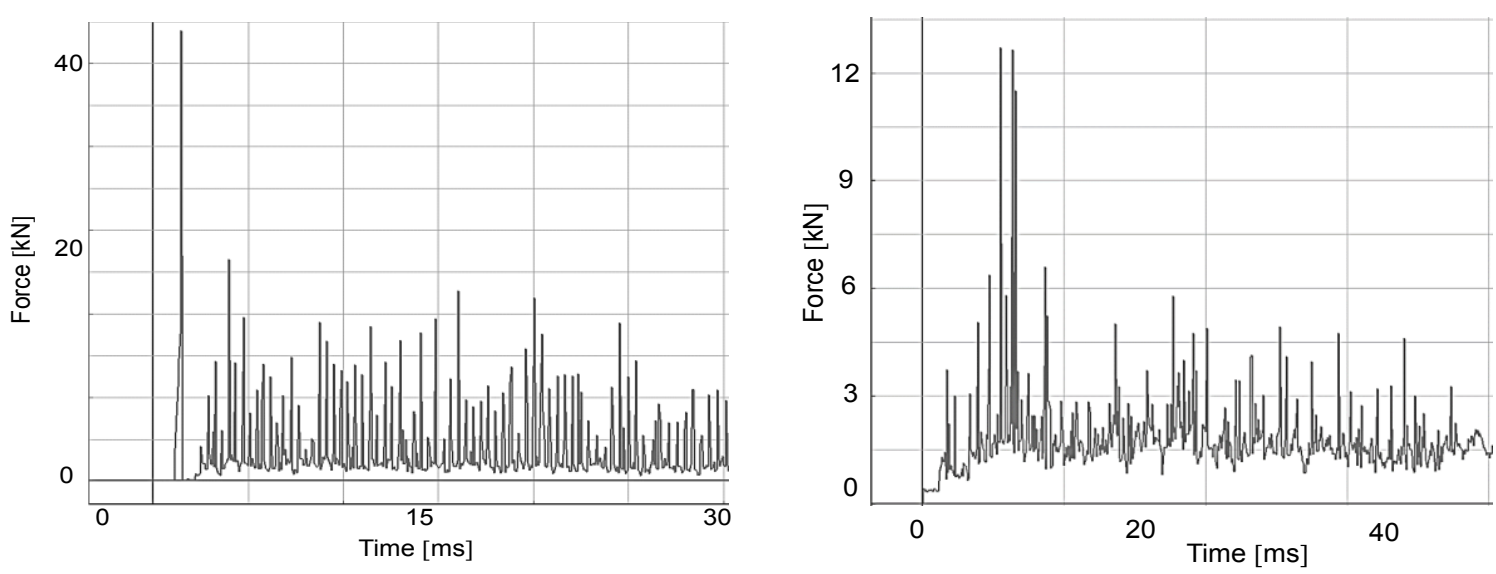

Figure 3: Force-deformation of axial loading (left), Force-deformation of 3-point-bending (right)

It is clearly visible that the maximum force is at a higher level than the average force. In the case of metal or aluminum the ratio of the maximal and average force is not very large. Fig. 3 (right) shows a force-deformation diagram of composite material and cylinder profile by 3-point bending. The graphic above represents a various tendency of two force sequences. The first power peak comes after a few 
milliseconds. The main difference between these two crash scenarios is the failure modes where in the case of an axial load, so-called crushing, many fibers break. With 3-point bending, crushing is impossible because the whole structure is merely bent. Nevertheless, the task of the structure is to absorb as much energy as possible and to achieve low deformation.

\subsection{Structure geometry}

There are two different geometry objects discussed in this article, a cylinder with an outside diameter of $\mathrm{d}_{\mathrm{a}}=150 \mathrm{~mm}$ and a rectangular with sides of $160 \times 80 \mathrm{~mm}$. The length of both structures is $500 \mathrm{~mm}$ (Fig. 4). The weight of all the variants is identical, only the wall thickness is a variable. Wall thickness was designed depending on the density of each material. For example, the wall thickness of the composite cylinder structure is at $3.8 \mathrm{~mm}$ and the wall thickness of the metal structure is at $1.2 \mathrm{~mm}$. For hybrid structures only aluminum is used, the metal layer is modeled externally at $1 \mathrm{~mm}$.
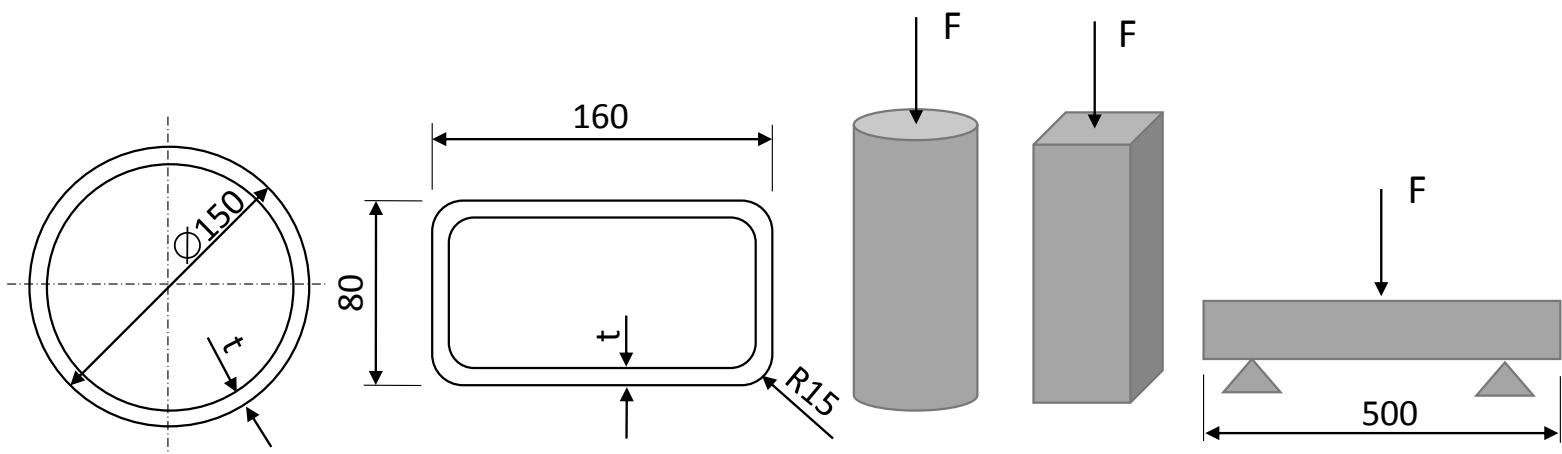

Figure 4: Structure geometry and load cases

All these combinations were modeled in Catia V5 and further processed in PAM-Crash. The composite structure was made with progressive crushing and delamination, using triggers and several shells as composite components. Fig. 5 shows the crushing process of a rectangle profile as a composite (left), the folding process of metal (middle) and a mixed process of a hybrid material during axial loading. It is visible that they show completely different failure characteristics as they play a very important role in energy absorption.
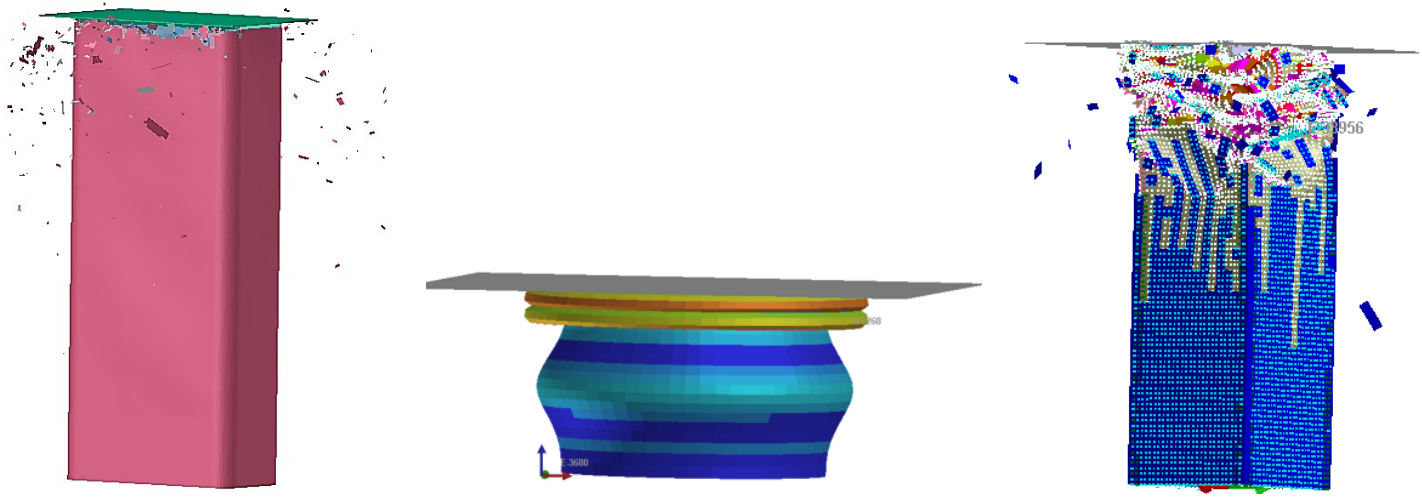

Figure 5: Crushing Process, composite (left), folding process of aluminum (middle), during axial loading, hybrid structure (right) 


\section{Applying the COPRAS Method}

Fig. 6 introduces the mathematical steps of COPRAS. The ranking is achieved by the creation of a decision matrix combining given alternatives and selection criteria.

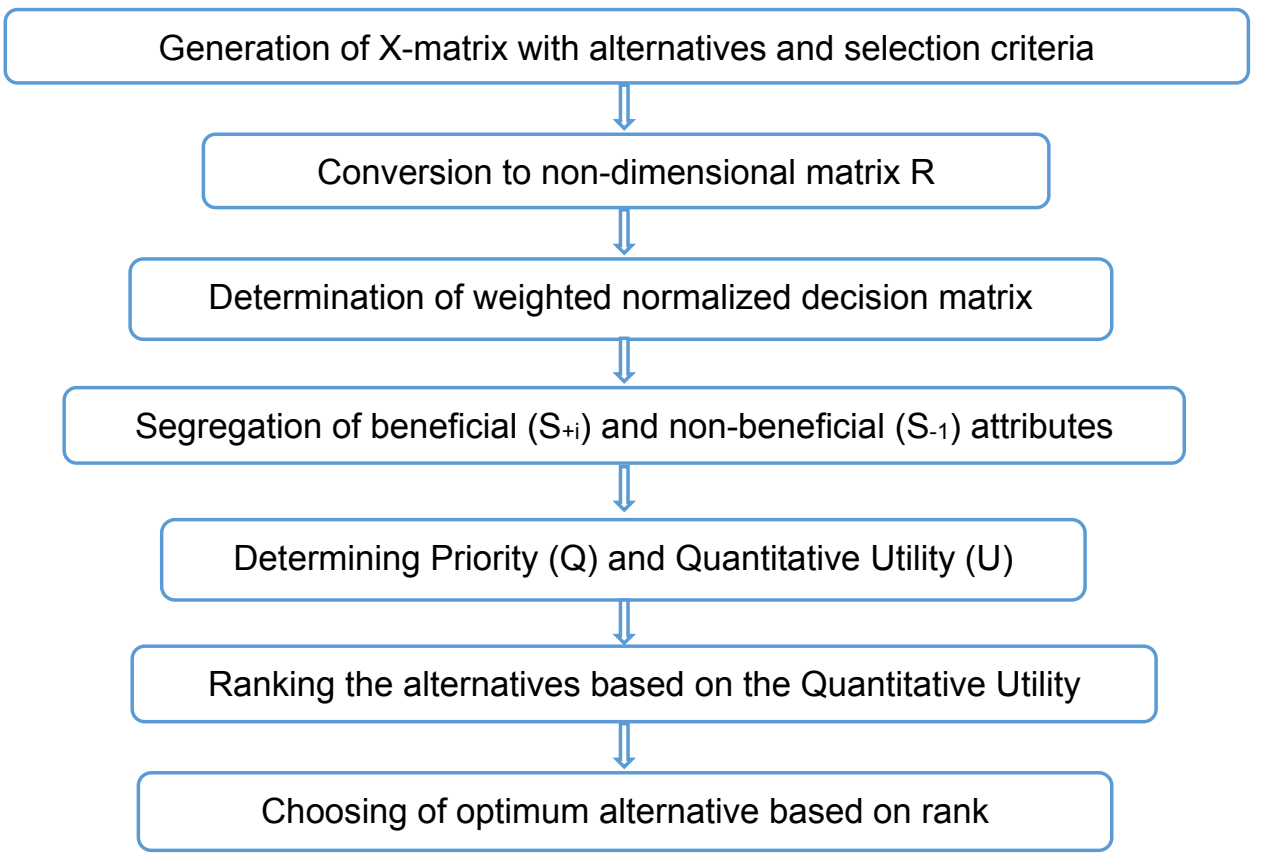

Figure 6: Mathematical steps in the Complex Proportional Assessment method

Table 1: Simulation results of axial loading

\begin{tabular}{lcccc}
\hline & EA [J] & $F_{\max }[\mathbf{k N}]$ & $F_{\text {avg }}[\mathbf{k N}]$ & D [mm] \\
\hline AR_1 & 1,200 & 300 & 20 & 30 \\
SR_1 & 1,200 & 1,200 & 2 & 20 \\
HR_045_1 & 3,100 & 210 & 15 & 60 \\
HR_45_1 & 4,200 & 50 & 10 & 80 \\
CR_45_1 & 2,300 & 95 & 15 & 140 \\
CR_045_1 & 2,200 & 64 & 15 & 160 \\
AO_1 & 1,300 & 350 & 25 & 20 \\
SO_1 & 1,350 & 1,300 & 4 & 25 \\
HO_045_1 & 2,200 & 410 & 20 & 60 \\
HO_45_1 & 2,400 & 330 & 25 & 30 \\
CO_45_1 & 4,000 & 43 & 5 & 270 \\
CO_045_1 & 3,900 & 60 & 12 & 200 \\
\hline
\end{tabular}

The impact at axial and 3-Point bending has been simulated with different materials. Table 1 provides an overview of the simulations. This table includes energy absorption $(E A)$, maximum force $\left(F_{\max }\right)$, average force $\left(\mathrm{F}_{\text {avg }}\right)$ and displacement $(\mathrm{D})$. The axial loading is given as an example. These parameters have been converted into specific energy absorption (SEA) and crash force efficiency (CFE). 
Load non-uniformity (LU) is calculated from the load fluctuation and average force. This is a key to better understanding the structures in the table. Steel $(S)$, aluminum $(A)$, composite $(C)$, hybrid $(H)$, cylinder $(\mathrm{O})$, rectangular $(\mathrm{R})$, axial loading (1), 3-Point-bending (2).

Table 2: X-Matrix, performance parameter of axial loading

\begin{tabular}{lcccc}
\hline & SEA [kJ/kg] & LU [-] & CFE [-] & D [mm] \\
\hline AR_1 & 15.3 & 0.13 & 0.07 & 30 \\
SR_1 & 30 & 0.0016 & 0.002 & 20 \\
HR_045_1 & 19.9 & 0.07 & 0.07 & 60 \\
HR_45_1 & 20.2 & 0.48 & 0.2 & 80 \\
CR_45_1 & 6.3 & 0.47 & 0.16 & 140 \\
CR_045_1 & 5.3 & 0.62 & 0.23 & 160 \\
AO_1 & 16 & 0.1 & 0.08 & 20 \\
SO_1 & 17.2 & 0.01 & 0.02 & 25 \\
HO_045_1 & 14.1 & 0.12 & 0.05 & 60 \\
HO_45_1 & 30.7 & 0.16 & 0.05 & 30 \\
CO_45_1 & 4.4 & 0.39 & 0.12 & 270 \\
CO_045_1 & 7.5 & 0.5 & 0.20 & 200 \\
\hline
\end{tabular}

The specific energy absorption refers to the weight of the crashed structure. For this reason, the energy absorption, which came from the simulation, is divided by the weight, which is constant. The data from the table above is converted into a non-dimensioned matrix $\mathrm{R}$ by normalizing the values of the different criteria. Further, based on the importance of the selection criteria weightings are given by comparing individual selections. The number of selection $(\mathrm{N})$ is calculated as follow: $N=n(n-1) / 2$; where $n$ is the number of selection criteria (Table 3). The pairs of criteria are subsequently compared to each other. The criterion which is most important is given a score of 3 , whereas the criterion which is least important is given a score of 1 . If both criteria are of equal importance, a score of 2 is given. For the weighting, EA was evaluated more important than load non-uniformity and displacement.

Table 3: D-Matrix

Number of selection sets $N=\frac{n(n-1)}{2}=4 \cdot \frac{3}{2}=6$

\begin{tabular}{lllllllrr} 
Selection criteria & $\mathbf{1}$ & $\mathbf{2}$ & $\mathbf{3}$ & $\mathbf{4}$ & $\mathbf{5}$ & $\mathbf{6}$ & $\mathbf{W j}$ & $\mathbf{w j}$ \\
\cline { 2 - 10 } & 3 & 2 & 3 & & & & 8 & 0.333333333 \\
Energy Absorption (EA) & 1 & & & 1 & 2 & & 4 & 0.166666667 \\
Load non-uniformity (LU) & 1 & 2 & & 3 & & 2 & 7 & 0.291666667 \\
Crush force efficiency (CFE) & & & 1 & & 2 & 2 & 5 & 0.208333333 \\
Displacement (D) & & & & & & & $\mathbf{2 4}$ & 1 \\
\end{tabular}

Furthermore, EA is considered to be equally important as CFE. Load non-uniformity is less important than CFE and as important as displacement. After the computation of weights, the weighted normalized decision matrix $\mathrm{D}$ is computed by multiplying the weights to the non-dimensioned R-matrix. Subsequently, the beneficial and non-beneficial attributes from the D-matrix are segregated (1-2). Load non-uniformity (LU) is a non-beneficial criterion where a lower value is required. While Energy Absorption and the Crush force efficiency index are beneficial criteria for which a higher value is required. 


$$
\begin{aligned}
& S_{+}=\sum_{i=1}^{m} S_{+i}=\sum_{i=1}^{m} \sum_{j=1}^{n} y_{+i j} \\
& S_{-}=\sum_{i=1}^{m} S_{-i}=\sum_{i=1}^{m} \sum_{j=1}^{n} y_{-i j}
\end{aligned}
$$

The priorities of the design concepts are calculated based on the notion of relative significance $\left(Q_{i}\right)$. The greater the value, the higher the priority of the structure. The design concept with the maximum relative significance $Q_{\max }$ is the best choice in the concept selection. The relative significance has been formulated as below:

$$
Q_{i}=S_{+i}+\frac{S_{-\min } \sum_{i=1}^{m} S_{-i}}{S_{-i} \sum_{i=1}^{m}\left(S_{-\min } / S_{-i}\right)}
$$

The quantitative utility value is directly related to the relative significance value. It equals the ratio of the individual relative significance value $Q i$ to the maximum relative significance value $Q_{\max }$. The utility value of 100 is considered to be the best choice. The quantitative utility is formulated as follows:

$$
U_{i}=\left[\frac{Q_{i}}{Q_{\max }}\right] \times 100 \%
$$

Based on the utility values, the evaluation takes into consideration all possible material and geometries. The results of the axial loading cases can be seen on the left and the 3-point bending case on the right side of Table 4.

Table 4: Ranking of different structures and load cases (axial-left), 3-point-bending (right)

\begin{tabular}{lllllll}
\hline & $\mathbf{Q}$ & $\mathbf{U}$ & Rank & $\mathbf{Q}$ & $\mathbf{U}$ & Rank \\
\hline AR_1/2 & 0.065114 & 68.612432 & 12 & 0.12282 & 59.11897 & 10 \\
SR_1/2 & 0.086001 & 90.621357 & 6 & 0.15443 & 74.33026 & 6 \\
HR_045_1/2 & 0.094901 & 100.000000 & 1 & 0.17835 & 85.84281 & 4 \\
HR_45_1/2 & 0.093449 & 98.469707 & 3 & 0.17075 & 82.18778 & 5 \\
CR_45_1/2 & 0.070528 & 74.317029 & 10 & 0.11886 & 57.20940 & 11 \\
CR_045_1/2 & 0.081172 & 85.532700 & 9 & 0.19319 & 92.98748 & 2 \\
AO_1/2 & 0.082920 & 87.374963 & 7 & 0.13843 & 66.63160 & 8 \\
SO_1/2 & 0.082920 & 87.374963 & 7 & 0.15008 & 72.23868 & 7 \\
HO_045_1/2 & 0.067595 & 71.226894 & 11 & 0.19150 & 92.17550 & 3 \\
HO_45_1/2 & 0.093616 & 98.645232 & 2 & 0.20776 & 100.00000 & 1 \\
CO_45_1/2 & 0.090275 & 95.125581 & 5 & 0.05362 & 25.81061 & 12 \\
CO_045_1/2 & 0.091509 & 96.425404 & 4 & 0.13006 & 13.67464 & 11 \\
\hline
\end{tabular}

Some parameters vary drastically, as is evident from Table 4 . The usability of a material depends on the load cases and other requirements. In our example, a crash case and three different material structures were taken into consideration. All the structures had the same weight with differing geometries. Therefore the wall thickness varied. As a result, the performances of all the presented alternatives were compared. In this article, no development process was introduced but a comparison 
of the part properties by the COPRAS-Method was presented. Depending on the boundary conditions, various usages of this method can be applied.

\section{Conclusion}

This article presents the COPRAS method with the aid of the axially and radially loaded structures of different materials. The important parameters are gained from the simulation. That method allows for a relative comparison of all variants for the application. The weighting in the third step plays a decisive role. Therefore, the operator must be aware of the importance in the paired comparison of individual parameters. At the end of the methodological procedure, a ranking sorts the appropriate applicability for the user. In case of an axial load, the rectangular structure as a hybrid with $0^{\circ}, \pm 45^{\circ}$ fiber orientation has the best performance. The hybrid structure with $\pm 45^{\circ}$ fiber orientation as a cylinder profile gave the second best performance, with $2 \%$ less in the ranking. The worst performance was achieved by the aluminum structure. Here, the quantitative utility has reached only $68 \%$. For radial loading, the best variation was shown by a hybrid structure as a cylinder profile and $\pm 45^{\circ}$ fiber orientation. The worst ranking was reached by a composite with $\pm 45^{\circ}$ ( $26 \%$ of quantitative utility). The purpose of this paper was not to assess the potential of the individual structures but to present the functions of the COPRAS method. In conclusion, there is a great possibility of high-quality support for the conceptual phase of the development, provided the criteria are selected correctly and subsequently weighted.

\section{References}

[1] Zavadskas, E. K.; Kaklauskas, A.; Turskis, Z.; Tamasaitiene, J.: Selection of the effective dwelling house walls by applying attributes values determined at intervals. J. of Civ. Eng. and Manag., 14(2) (2008), pp. 85-93. doi: 10.3846/1392-3730.2008.14.3

[2] Gadakh, V. S.: Application of complex proportional assessment method for vendor selection. Int. J. of Log. Res. and Appl., 17(1) (2013), pp. 23-34. doi: 10.1080/13675567.2013.836159

[3] Chatterjeea, P.; Athawale, V. M.; Chakraborty, S.: Materials selection using complex proportional assessment and evaluation of mixed data methods. Mat. and Des., 32(2) (2011), pp. 851-860. doi: 10.1016/j.matdes.2010.07.010

[4] Vahdani, B.; Mousavi, S. M.; Tavakkoli-Moghaddam, R.; Ghodratnama, A.; Mohammadi, M.: Robot selection by a multiple criteria complex proportional assessment method under an intervalvalued fuzzy environment. Int. J. of Adv. Manuf. Technol., 53(5-8) (2014), pp. 687-697. doi:10.1007/s00170-014-5849-9

[5] VDI-Richtlinie 2014: Entwicklung von Bauteilen aus Faser-Kunststoff-Verbund. Blatt 2: Konzeption und Gestaltung. Berlin: Beuth, 1993.

[6] Handbuch Faserverbundkunststoffe/Composites. Wiesbaden: Springer, 2013.

[7] Huang, M. Y.; Tai, Y.-S.; Hu, H. T.: Numerical study on hybrid tubes subjected to static and dynamic loading. Appl. Composite Mat., 19(1) (2012), pp. 1-19. doi:10.1007/s10443-010-9176-5

[8] Farley, G. L.: Effect of fiber and matrix maximum strain on the energy-absorption of compositematerials. J. of Composite Mat., 20(4) (1986), pp. 322-334. doi:10.1177/002199838602000401

[9] Mejlej, V. G.; Osorio, D.; Vietor, T.: An improved fatigue failure model for multidirectional fiberreinforced composite laminates under any stress ratios of cyclic loading. Procedia CIRP, 66 (2017), pp. 27-32. doi:10.1016/j.procir.2017.03.303

[10] Mejlej, V. G.; Falkenberg, P.; Türck, E., Vietor, T. (in press): Multidisciplinary structural optimization using of NSGA-II and $\varepsilon$-Constraint method in lightweight application. In: Schumacher, A. et al. (eds.): Advances in Structural and Multidisciplinary Optimization, Cham: Springer, 2018. 